\title{
Heterosis for some physio-morphological plant traits in spring wheat crosses
}

\author{
Shahzadi Mahpara ${ }^{1 *}$, Muhammad Ishaq Asif Rehmani ${ }^{2}$, Safdar Hussain $^{2}$, \\ Javaid Iqbal ${ }^{2}$, Muhammad Kamran Qureshi ${ }^{3}$, Muhammad Ahmed \\ Shehzad ${ }^{4}$ and Javed Shabbir Dar ${ }^{5}$ \\ 1. Department of Plant Breeding and Genetics, Ghazi University, D.G. Khan-Pakistan \\ 2. Department of Agronomy, Ghazi University, D.G. Khan-Pakistan \\ 3. Department of Plant Breeding and Genetics, Bahauddin Zakariya University, Multan-Pakistan \\ 4. Department of Statistics, Bahauddin Zakariya University, Multan-Pakistan \\ 5. Department of Agronomy, SZABAC Dokri, Larkana-Pakistan \\ *Corresponding author's email: mahpara.gudgk@gmail.com \\ Citation \\ Shahzadi Mahpara, Muhammad Ishaq Asif Rehmani, Safdar Hussain, Javaid Iqbal, Muhammad Kamran Qureshi, \\ Muhammad Ahmed Shehzad and Javed Shabbir Dar. 2017. Heterosis for some physio-morphological plant traits in \\ spring wheat crosses. Pure and Applied Biology. Vol. 6, Issue 4, pp1103-1110. \\ http://dx.doi.org/10.19045/bspab.2017.600118
}

Received: 20/04/2017 Revised: 30/08/2017

Accepted: 06/09/2017

Online First: 09/09/2017

\section{Abstract}

An experiment was conducted at the experimental site of Department of Plant Breeding and Genetics, University of Agriculture, Faisalabad, Pakistan to study heterosis analysis and gene action on seven wheat varieties and their $\mathrm{F}_{1}$ hybrids in $7 \times 7$ diallel fashion. Data for flag leaf area, flag leaf related traits including epidermal cell size, stomatal frequency, leaf venation, stomatal size, hygrophilic colloids and grain yield per plant was collected and analyzed. Higher positive values for flag leaf area (16.53) and leaf venation (9.89) were found in MH-97 × 4072. Negative hygrophilic colloids (-28.00) and stomatal frequency (-13.92), however, positive flag leaf area (9.35) and leaf venation (5.62) were recorded for Uqab-2000 × Punjab-96 with, and. In contrast, $4072 \times$ Punjab-96 produced positive leaf venation (8.36) and epidermal cell size (2.36), but negative stomatal frequency (-8.18), stomatal size (-3.65). Considering genetic behavior of these three crosses, they should be utilized for future wheat breeding program to develop varieties able to survive not only under optimum environmental conditions but also perform better under stressful environment.

Keywords: $F_{1}$ hybrids; Flag leaf; Flag leaf related traits; Gene action; Heterosis; Wheat

\section{Introduction}

In Pakistan wheat (Triticum aestivum L.) is staple food and an important winter cereal crop and its demand is gradually increasing due to increasing population [1-3]. In Pakistan wheat was cultivated on an area of 9.26 million hectare ( $\mathrm{m} \mathrm{ha}$ ) during 2015-16, producing 25.45 million tons of wheat [4]. Leaf is involved in the photosynthetic process in all green plants and it has the main role in the production photosynthates and dry matter of crop plants [5]. So increased leaf area increases the rate of photosynthetic process in plants and provides increased translocation of photosynthates in spikes during grain filling stages ultimately, the yield of the plant will increase $[6,7]$. In wheat, flag leaf area has significant contributions in improving grain yield [8]. Consequently, flag leaf and its 
related traits also contribute to enhance yield of newly developed wheat varieties like less number of stomata and small size of stomata because these traits help the plant to use water efficiently $[9,10]$. Geneticists remained fascinated by heterosis even after 100 years [11]. Shull [12] rediscovered heterosis in maize and described it as "heterosis is an increased vigor, growth, size, rate of development and other desirable characteristics of hybrids as compared with related parents". Hybrid vigor or heterosis is dissimilarity due to cross combinations and the mean of both parents [13]. It is generally termed as mid-parent heterosis. Utilization of heterosis is not only applied for crosspollinated crops in $\mathrm{F}_{1}$ hybrids for high yield on large scale but its heterotic effects are also available in self-pollinated crops like wheat as well. In wheat Freeman [14] first time described heterosis when he found taller $F_{1}$ than its tall parents. Commercial utilization of heterosis was suggested by Briggle [15]. It is observed by many researchers that positive heterosis is important in some plant traits like grain yield per plant $[16,17]$ flag leaf area $[18$, 19]. This study was performed to evaluate $F_{1}$ hybrids of seven wheat varieties for flag leaf area and its related traits using various biometrical tools and to explore new genetic potential among available genetic resources in the country. Produced new combinations can further be utilized in the future breeding program to enhance existing yield level.

\section{Materials and methods}

An experiment was executed at experimental site of Plant Breeding and Genetics Department, University of Agriculture, Faisalabad, Pakistan. Soil of experimental site had $\mathrm{pH} 6.5$ and $\mathrm{EC}$ of 4 $\mathrm{dS} / \mathrm{m}$. The complete diallel analysis was adopted in which seven wheat varieties/ lines were sown in the field and were hybridized at anthesis to develop new genetic combinations for hereditary study. At maturity seeds from crosses were collected and were sown next year along with parent varieties under triplicated randomized complete block design in field conditions. Every replication contained thirty plants per genotype having all the parents and $F_{1}$ hybrids. Distance for rows and for plants was kept $30 \mathrm{~cm}$ and $15 \mathrm{~cm}$. Local recommendations including agronomic practices and crop protection measures were uniformly applied throughout the growing period to avoid any stress. Data for leaf venation, flag leaf area, stomatal size, the number of stomata, epidermal cell size, hygrophillic colloids and grain yield per plant at maturity were recorded and used for analysis. Variances of data collected as per replications for all genotypes and their hybrids were subjected according to statistically analysis using DMRT following Steel et al. [20] and then it was further subjected for calculating heterosis and heterobeltiosis as described earlier [21].

\section{Results}

\section{Analysis of variance}

Data collected for plant characters were subjected to find out significant differences among 49 genotypes through ANOVA as suggested by Steel et al. [20]. All genotypes were significantly different for studied traits except stomatal size (Table 1). The significant genotypic differences permitted further genetic analysis following the technique suggested by Hayman [22] Hayman [23] and Jinks [24].

\section{Heterosis analysis}

For flag leaf trait, positive heterosis was observed in 11 crosses (Table 2). Significantly higher heterosis and heterobeltosis for flag leaf area was showed by only one hybrid i.e., MH-97 × 4072 . 
Table 1. Mean squares of various plant traits in $7 \times 7$ diallel crosses of Triticum aestivum $\mathrm{L}$.

\begin{tabular}{|l|l|l|l|l|l|l|l|l|}
\hline Source & DF & $\begin{array}{l}\text { Flag leaf } \\
\text { area }\end{array}$ & $\begin{array}{l}\text { Leaf } \\
\text { venation }\end{array}$ & $\begin{array}{l}\text { Stomatal } \\
\text { frequency }\end{array}$ & $\begin{array}{l}\text { Stomatal } \\
\text { size }\end{array}$ & $\begin{array}{l}\text { Epidermal } \\
\text { cell size }\end{array}$ & $\begin{array}{l}\text { Hygrophilic } \\
\text { colloids }\end{array}$ & $\begin{array}{l}\text { Grain yield } \\
\text { per plant }\end{array}$ \\
\hline Replication & 2 & 4.21 & 1.02 & 2477 & 7002 & 1003 & 0.00017 & 2.23 \\
\hline Genotypes & 48 & $21.77 * *$ & $16.59^{* *}$ & $5919^{* *}$ & $1394 \mathrm{~ns}$ & $5274 * *$ & $0.0235^{* *}$ & $9.12^{* *}$ \\
\hline Error & 96 & 2.25 & 4.14 & 2460 & 9444 & 1025 & 0.0096 & 3.08 \\
\hline Mean & & 15.184 & 36.398 & 575.710 & 1782.14 & 3697.95 & 0.164 & 20.043 \\
\hline CV \% & & 9.873 & 5.589 & 8.616 & 5.453 & 8.659 & 28.89 & 8.764 \\
\hline
\end{tabular}

**, Significant at $P \leq 0.01 ; *$, Significant at $P \leq 0.05 ; \mathrm{ns}$, Non-significant at $P>0.05$.

Whereas rest of the crosses displayed a reduction in flag leaf area as compared to their mid parents. Majority of crosses i.e., 37 out of 42 crosses exhibited negative heterobeltosis. Heterosis for leaf venation (Table 2) showed that 23 crosses exhibited an increase in leaf venation over mid-parent values whereas only 6 crosses gave significant values. Highest positive heterosis was observed in Iqbal-2000 $\times$ Uqab-2000 (10.23) and MH-97 × 4072 (9.89). The maximum positive heterobeltiosis for leaf venation was recorded for $4072 \times$ Parwaz94 (10.26), similarly other 12 crosses also showed positive heterobeltosis.

Heterosis observed for stomatal frequency revealed that 28 crosses showed decreased stomatal frequency over mid parent value out of which only 6 crosses were significant. Highest level of heterosis was observed in Uqab-2000 $\times$ Iqbal- 2000 (-16.78) and Uqab-2000 $\times$ Punjab-96 (-13.92). More than $75 \%$ of crosses i.e., 34 crosses out of 42 crosses produced negative heterobeltosis, from which heterobeltosis reached significant level in 15 crosses for stomatal frequency. Surprisingly maximum heterobeltosis was found in the crosses with maximum values of negative heterosis.

In case of stomatal size approximately half crosses i.e., 23 crosses displayed increased size of stomata, however stomatal size was decreased in 19 crosses (Table 2). Maximum reduction in stomatal size was recorded in MH-97 × Iqbal-2000 (4.32). Heterobeltosis for stomatal size explained that 30 crosses out of 42 crosses showed a reduction in stomatal size, whereas maximum reduction was found in cross Punjab-96 × Iqbal-2000 (-7.56). Heterosis data for epidermal cell size revealed that 20 crosses showed positive heterosis however, only 5 of these were significantly positive. Highest value of heterosis for epidermal cell size was recorded in $\mathrm{MH}-97 \times$ Punjab-96 (25.66) followed by Parwaz-94 $\times$ Uqab-2000 (15.32). One out of eight crosses showed significant positive heterobeltosis which was observed in the crosses with maximum positive heterosis values. While 34 crosses showed reduced size of epidermal cells. Similarly, 13 crosses displayed decreased hygrophilic colloids value for heterosis. Moreover, 11 out of 28 crosses demonstrated significantly decreased heterosis, while 12 crosses showed positive heterosis for hygrophilic colloids. Maximum decrease in hygrophillic colloids was observed in MH-97 × Uqab-2000 (-81.67) followed by Uqab-2000 $\times$ Iqbal-2000 (75.0) and Iqbal-2000 $\times$ Parwaz-94 (-72.85). Similarly, maximum negative heterobeltosis was also found in crosses with positive heterosis for hygrophilic colloids. Considering decreasing hygrophilic colloids of Uqab-2000 and Iqbal -2000, both proved to be best parents. For grain yield parameter, 20 crosses exhibited increased trend for midparent value of which only 2 were important. Greatest positive heterosis value was observed in $4072 \times$ Punjab-96 (21.95). However increased heterobeltosis was present in 7 cross combinations in which one hybrid was prominent with the highest heterobeltosis (11.33). 
Mahapara et al.

Table 2. Heterosis (\%) and heterobeltiosis (\%) effects in flag leaf are and its related traits in $7 \times 7$ diallel crosses

\begin{tabular}{|c|c|c|c|c|c|c|c|c|c|c|c|c|c|c|}
\hline \multirow[t]{2}{*}{ Crosses } & \multicolumn{2}{|c|}{ Flag leaf area } & \multicolumn{2}{|c|}{ Leaf venation } & \multicolumn{2}{|c|}{$\begin{array}{c}\text { Stomatal } \\
\text { frequency }\end{array}$} & \multicolumn{2}{|c|}{ Stomatal size } & \multicolumn{2}{|c|}{ Epidermal cell size } & \multicolumn{2}{|c|}{ Hygrophilic colloids } & \multicolumn{2}{|c|}{ Grain yield per plant } \\
\hline & $\mathrm{Ht}$ & $\mathrm{Hb}$ & $\mathrm{Ht}$ & $\mathrm{Hb}$ & $\mathrm{Ht}$ & $\mathrm{Hb}$ & $\mathrm{Ht}$ & $\mathrm{Hb}$ & $\mathrm{Ht}$ & $\mathrm{Hb}$ & $\mathrm{Ht}$ & $\mathrm{Hb}$ & $\mathrm{Ht}$ & $\mathrm{Hb}$ \\
\hline $\begin{array}{c}\text { Shahkar-95 } \times \\
\text { Parwaz-94 }\end{array}$ & $-9.24^{\mathrm{NS}}$ & $-15.18^{\mathrm{NS}}$ & $-9.27 * *$ & $\begin{array}{c}- \\
11.19 * *\end{array}$ & 4.10 & $-1.14^{\mathrm{NS}}$ & $\begin{array}{c}- \\
2.75^{\mathrm{NS}}\end{array}$ & $\begin{array}{c}- \\
3.91^{\mathrm{NS}}\end{array}$ & $-3.95^{\mathrm{NS}}$ & $-4.83^{\mathrm{NS}}$ & $-0.99^{\mathrm{NS}}$ & $-28.57^{\mathrm{NS}}$ & $-1.93^{\mathrm{NS}}$ & $-2.60^{\mathrm{NS}}$ \\
\hline $\begin{array}{c}\text { Shahkar-95 × Iqbal- } \\
2000\end{array}$ & $-20.17 * *$ & $-38.78 * *$ & $-9.25 * *$ & $\begin{array}{c}- \\
12.97 * *\end{array}$ & 9.62 & $5.01^{\mathrm{NS}}$ & $3.08^{\mathrm{NS}}$ & $0.74^{\mathrm{NS}}$ & $-9.25^{\mathrm{NS}}$ & $-13.94 *$ & $-53.85 * *$ & $-68.42 * *$ & $0.12^{\mathrm{NS}}$ & $-8.91^{\mathrm{NS}}$ \\
\hline $\begin{array}{c}\text { Shahkar-95 × Uqab- } \\
2000\end{array}$ & $2.63^{\mathrm{NS}}$ & $-5.49^{\mathrm{NS}}$ & $-4.65^{\mathrm{NS}}$ & $-5.99^{\mathrm{NS}}$ & 3.34 & $-5.39^{\mathrm{NS}}$ & $\begin{array}{c}- \\
1.94^{\mathrm{NS}}\end{array}$ & $-7.43 *$ & $3.12^{\mathrm{NS}}$ & $-7.10^{\mathrm{NS}}$ & $-13.33^{\mathrm{NS}}$ & $-25.71^{\mathrm{NS}}$ & $1.44^{\mathrm{NS}}$ & $-3.61^{\mathrm{NS}}$ \\
\hline Shahkar-95 × MH-97 & $-7.81^{\mathrm{NS}}$ & $-19.34 * *$ & $-3.18^{\mathrm{NS}}$ & $-8.25^{*}$ & 4.04 & $2.59^{\mathrm{NS}}$ & $0.84^{\mathrm{NS}}$ & $0.62^{\mathrm{NS}}$ & $4.00^{\mathrm{NS}}$ & $1.41^{\mathrm{NS}}$ & $33.33^{\mathrm{NS}}$ & $14.29^{\mathrm{NS}}$ & -0.93 & $-4.20^{\mathrm{NS}}$ \\
\hline Shahkar-95 × 4072 & $-1.37^{\mathrm{NS}}$ & $-17.88 *$ & $1.48^{\mathrm{NS}}$ & $-2.78^{\mathrm{NS}}$ & -0.51 & $-2.00^{\mathrm{NS}}$ & $0.58^{\mathrm{NS}}$ & $0.21^{\mathrm{NS}}$ & $-2.30^{\mathrm{NS}}$ & $-12.38^{*}$ & $-31.87^{\mathrm{NS}}$ & $-55.71^{\mathrm{NS}}$ & $1.04^{\mathrm{NS}}$ & $2.46^{\mathrm{NS}}$ \\
\hline $\begin{array}{c}\text { Shahkar-95 × Punjab- } \\
96\end{array}$ & $-2.01^{\mathrm{NS}}$ & $-24.39 * *$ & $0.82^{\mathrm{NS}}$ & $-1.65^{\mathrm{NS}}$ & 1.52 & $-2.02^{\mathrm{NS}}$ & $3.58^{\mathrm{NS}}$ & $1.72^{\mathrm{NS}}$ & $1.96^{\mathrm{NS}}$ & $-0.40^{\mathrm{NS}}$ & $0.00^{\mathrm{NS}}$ & $-14.29^{\mathrm{NS}}$ & $-8.42^{\mathrm{NS}}$ & $-19.62 * *$ \\
\hline $\begin{array}{c}\text { Parwaz-94 } \times \text { Shahkar- } \\
95\end{array}$ & $-2.62^{\mathrm{NS}}$ & $-9.00^{\mathrm{NS}}$ & $-1.90^{\mathrm{NS}}$ & $-3.98^{\mathrm{NS}}$ & -9.64 & $\begin{array}{c}- \\
14.19 * *\end{array}$ & $1.02^{\mathrm{NS}}$ & $\begin{array}{c}- \\
0.17^{\mathrm{NS}}\end{array}$ & $3.86^{\mathrm{NS}}$ & $-2.91^{\mathrm{NS}}$ & $18.81^{\mathrm{NS}}$ & $-14.29^{\mathrm{NS}}$ & $-4.03^{\mathrm{NS}}$ & $-4.95^{\mathrm{NS}}$ \\
\hline $\begin{array}{c}\text { Parwaz-94 × Iqbal- } \\
2000 \\
\end{array}$ & $-36.08 * *$ & $-48.41 * *$ & $-4.50^{\mathrm{NS}}$ & $-6.49^{\mathrm{NS}}$ & 2.38 & $-6.63^{\mathrm{NS}}$ & $0.33^{\mathrm{NS}}$ & $\begin{array}{c}- \\
0.77^{\mathrm{NS}} \\
\end{array}$ & $-0.26^{\mathrm{NS}}$ & $-6.23^{\mathrm{NS}}$ & $-63.80 * *$ & $-78.95 * *$ & $-11.72 *$ & $-19.19 * *$ \\
\hline $\begin{array}{c}\text { Parwaz-94 } \times \text { Uqab- } \\
2000 \\
\end{array}$ & $2.60^{\mathrm{NS}}$ & $0.99^{\mathrm{NS}}$ & $-2.35^{\mathrm{NS}}$ & $-5.72^{\mathrm{NS}}$ & -1.80 & $-5.54^{\mathrm{NS}}$ & $\begin{array}{c}- \\
0.01^{\mathrm{NS}} \\
\end{array}$ & $\begin{array}{c}- \\
4.53^{\mathrm{NS}} \\
\end{array}$ & $15.32 * *$ & 4.74 & $23.46^{\mathrm{NS}}$ & $0.00^{\mathrm{NS}}$ & $0.09^{\mathrm{NS}}$ & $-4.27^{\mathrm{NS}}$ \\
\hline Parwaz-94 × MH-97 & $-5.79^{\mathrm{NS}}$ & $-12.26^{\mathrm{NS}}$ & $-9.48 * *$ & $12 . \overline{-} * *$ & 1.78 & $-2.01^{\mathrm{NS}}$ & $2.74^{\mathrm{NS}}$ & $1.28^{\mathrm{NS}}$ & $4.06^{\mathrm{NS}}$ & $2.40^{\mathrm{NS}}$ & $23.46^{\mathrm{NS}}$ & $0.00^{\mathrm{NS}}$ & $-9.93^{\mathrm{NS}}$ & $-13.47 *$ \\
\hline Parwaz-94 × 4072 & $-6.73^{N S}$ & $-17.67 * *$ & $-6.49^{N S}$ & $12.24 * *$ & 1.05 & $-2.66^{\mathrm{NS}}$ & $-\overline{-}$ & $\begin{array}{c}- \\
5.51^{\mathrm{NS}} \\
\end{array}$ & $-3.50^{\mathrm{NS}}$ & $-14.16^{*}$ & $-19.23^{\mathrm{NS}}$ & $-32.26^{\mathrm{NS}}$ & $0.57^{\mathrm{NS}}$ & $-3.09^{\mathrm{NS}}$ \\
\hline Parwaz-94 × Punjab-96 & $-17.05 * *$ & $-32.61 * *$ & $-7.06^{\mathrm{NS}}$ & $-7.40^{\mathrm{NS}}$ & -3.59 & $-11.48^{*}$ & $\begin{array}{c}- \\
2.36^{\mathrm{NS}}\end{array}$ & $\begin{array}{c}- \\
5.22^{\mathrm{NS}}\end{array}$ & $11.69 * \mathrm{NS}$ & $10.09^{\mathrm{NS}}$ & $25.93^{\mathrm{NS}}$ & $2.00^{\mathrm{NS}}$ & $2.36^{\mathrm{NS}}$ & $-9.63^{\mathrm{NS}}$ \\
\hline $\begin{array}{c}\text { Iqbal-2000 } \times \text { Shahkar- } \\
95\end{array}$ & $-18.22 * *$ & $-37.28 * *$ & $6.044^{\mathrm{NS}}$ & $1.68^{\mathrm{NS}}$ & -7.52 & $\begin{array}{c}-11.41 \\
\text { NS }\end{array}$ & $\begin{array}{c}- \\
1.34^{\mathrm{NS}}\end{array}$ & $\begin{array}{c}- \\
3.57^{\mathrm{NS}}\end{array}$ & $3.90^{\mathrm{NS}}$ & $-1.46^{\mathrm{NS}}$ & $-69.23 * *$ & $-78.95 * *$ & $\begin{array}{c}- \\
15.67 * *\end{array}$ & $-23.28 * *$ \\
\hline $\begin{array}{c}\text { Iqbal-2000 } \times \text { Parwaz- } \\
94 \\
\end{array}$ & $-3.49^{\mathrm{NS}}$ & $-22.11 * *$ & $2.47^{\mathrm{NS}}$ & $0.34^{\mathrm{NS}}$ & -5.65 & $-13.97 *$ & $\begin{array}{c}- \\
1.25^{\mathrm{NS}} \\
\end{array}$ & $\begin{array}{c}- \\
2.34^{\mathrm{NS}}\end{array}$ & -7.41 & $-12.96^{*}$ & $-72.85 * *$ & $-84.21 * *$ & $2.43^{\mathrm{NS}}$ & $-6.23^{\mathrm{NS}}$ \\
\hline $\begin{array}{c}\text { Iqbal-2000 } \times \text { Uqab- } \\
2000 \\
\end{array}$ & $-17.11 * *$ & $-32.27 * *$ & $10.23 * *$ & $4.29^{\mathrm{NS}}$ & -9.21 & $\begin{array}{c}- \\
20.06^{* *} \\
\end{array}$ & $3.78^{\mathrm{NS}}$ & $0.16^{\mathrm{NS}}$ & $9.39^{\mathrm{NS}}$ & $-5.99^{\mathrm{NS}}$ & $-33.33^{*}$ & $-57.89 * *$ & $1.35^{\mathrm{NS}}$ & $-3.20^{\mathrm{NS}}$ \\
\hline Iqbal-2000 × MH-97 & $-12.66^{* *}$ & $-25.24 * *$ & $-3.60^{\mathrm{NS}}$ & $-4.78^{\mathrm{NS}}$ & -5.39 & $-10.58^{\mathrm{NS}}$ & $0.16^{\mathrm{NS}}$ & $2.32^{-} \mathrm{NS}$ & $12.76^{* *}$ & $4.41^{\mathrm{NS}}$ & $-41.67 * *$ & $-63.16 * *$ & $-7.66^{\mathrm{NS}}$ & $-18.50 * *$ \\
\hline Iqbal-2000 × 4072 & $2.07^{\mathrm{NS}}$ & $-8.00^{\mathrm{NS}}$ & $4.78^{\mathrm{NS}}$ & $-3.55^{N S}$ & 1.67 & $-3.99^{\mathrm{NS}}$ & $4.38^{\mathrm{NS}}$ & $6.89^{-}$ & $-2.41^{\mathrm{NS}}$ & $-8.08^{\mathrm{NS}}$ & $-14.69^{\mathrm{NS}}$ & $-52.63 * *$ & $-7.17^{\mathrm{NS}}$ & $-11.99^{\mathrm{NS}}$ \\
\hline $\begin{array}{c}\text { Iqbal-2000 } \times \text { Punjab- } \\
96 \\
\end{array}$ & $\begin{array}{c}1.45^{\mathrm{NS}} \\
\mathrm{NS}\end{array}$ & $0.58^{\mathrm{NS}}$ & $2.77^{\mathrm{NS}}$ & $0.99^{\mathrm{NS}}$ & 4.39 & $3.59^{\mathrm{NS}}$ & $2.79^{\mathrm{NS}}$ & $\begin{array}{c}- \\
1.29^{\mathrm{NS}} \\
\end{array}$ & $9.22^{\mathrm{NS}}$ & $1.29^{\mathrm{NS}}$ & $-41.67^{\mathrm{NS}}$ & $-63.16 * *$ & $0.78^{\mathrm{NS}}$ & $-4.66^{\mathrm{NS}}$ \\
\hline $\begin{array}{c}\text { Uqab-2000 } \times \text { Shahkar- } \\
95\end{array}$ & $\begin{array}{c}9.02^{\mathrm{NS}} \\
\mathrm{NS}\end{array}$ & $0.39^{\mathrm{NS}}$ & $1.07^{\mathrm{NS}}$ & $-0.30^{\mathrm{NS}}$ & $\begin{array}{c}- \\
12.46^{* *}\end{array}$ & $\begin{array}{c}- \\
19.86^{* *} \\
\end{array}$ & $\begin{array}{c}- \\
0.81^{\mathrm{NS}} \\
\end{array}$ & $\begin{array}{c}- \\
6.36^{\mathrm{NS}} \\
\end{array}$ & $14.00 * *$ & $2.68^{\mathrm{NS}}$ & $-16.67^{\mathrm{NS}}$ & $-28.57^{\mathrm{NS}}$ & $-1.03^{\mathrm{NS}}$ & $-5.95^{\mathrm{NS}}$ \\
\hline $\begin{array}{c}\text { Uqab-2000 × Parwaz- } \\
94\end{array}$ & $-2.25^{\mathrm{NS}}$ & $-3.78^{\mathrm{NS}}$ & $-0.25^{\mathrm{NS}}$ & $-3.70^{\mathrm{NS}}$ & $-11.36^{*}$ & $\begin{array}{c}- \\
14.74 * *\end{array}$ & $7.34 *$ & $2.49^{\mathrm{NS}}$ & $3.04^{\mathrm{NS}}$ & $-6.41^{\mathrm{NS}}$ & $-1.23^{\mathrm{NS}}$ & $-20.00^{\mathrm{NS}}$ & $-10.66^{*}$ & $-14.55^{*}$ \\
\hline Uqab-2000 × Iqbal- & $-7.54^{\mathrm{NS}}$ & $-24.46 * *$ & $-7.09 *$ & - & - & - & $2.05^{\mathrm{NS}}$ & - & $-6.85^{\mathrm{NS}}$ & - & $-75.00 * *$ & $-84.21 * *$ & 4.92 & 0.20 \\
\hline
\end{tabular}


Pure Appl. Biol., 6(4):1103-1110, December, 2017 http://dx.doi.org/10.19045/bspab.2017.600118

\begin{tabular}{|c|c|c|c|c|c|c|c|c|c|c|c|c|c|c|}
\hline 2000 & & & & $12.10 * *$ & $16.78 * *$ & $26.73 * *$ & & $1.50^{\mathrm{NS}}$ & & $19.96 * *$ & & & & \\
\hline Uqab-2000 × MH-97 & $-7.35^{\mathrm{NS}}$ & $1.48^{\mathrm{NS}}$ & $-0.18^{\mathrm{NS}}$ & $-6.65^{\mathrm{NS}}$ & -2.92 & $-9.98 *$ & $1.75^{-} \mathrm{NS}$ & $-7.44^{*}$ & $5.86^{\mathrm{NS}}$ & $-2.42^{\mathrm{NS}}$ & $20.00^{\mathrm{NS}}$ & $20.00^{\mathrm{NS}}$ & 1.62 & -6.46 \\
\hline Uqab-2000 × 4072 & $4.79^{\mathrm{NS}}$ & $-6.20^{\mathrm{NS}}$ & $-3.74^{\mathrm{NS}}$ & $-6.52^{\mathrm{NS}}$ & -5.65 & $-12.42 *$ & $\begin{array}{c}- \\
0.66^{\mathrm{NS}}\end{array}$ & $\begin{array}{c}- \\
6.55^{\mathrm{NS}} \\
\end{array}$ & $-10.55^{*}$ & $\begin{array}{c}- \\
26.83^{* *}\end{array}$ & $12.68^{\mathrm{NS}}$ & $-20.00^{\mathrm{NS}}$ & -8.98 & -9.68 \\
\hline $\begin{array}{c}\text { Uqab-2000 } \times \text { Punjab- } \\
96 \\
\end{array}$ & $9.35^{\mathrm{NS}}$ & $\begin{array}{l}-10.05 * \\
\text { NS }\end{array}$ & $5.62^{\mathrm{NS}}$ & $1.61^{\mathrm{NS}}$ & $13.92 * *$ & $\begin{array}{c}- \\
23.70 * *\end{array}$ & $2.25^{\mathrm{NS}}$ & $5.09^{\mathrm{NS}}$ & $4.65^{\mathrm{NS}}$ & $-3.68^{\mathrm{NS}}$ & $-28.00^{\mathrm{NS}}$ & $-28.00^{\mathrm{NS}}$ & 6.05 & -2.50 \\
\hline MH-97 × Shahkar-95 & $7.42^{\mathrm{NS}}$ & $-6.02^{\mathrm{NS}}$ & $0.79^{\mathrm{NS}}$ & $-4.40^{\mathrm{NS}}$ & -4.52 & $-5.85^{\mathrm{NS}}$ & $2.25^{\mathrm{NS}}$ & $2.03^{\mathrm{NS}}$ & $\begin{array}{c}- \\
6.02^{\mathrm{NSNS}} \\
\end{array}$ & $-8.36^{\mathrm{NS}}$ & $-33.33^{\mathrm{NS}}$ & $-42.86^{\mathrm{NS}}$ & $\begin{array}{c}- \\
17.54 * * \\
\end{array}$ & $-20.26 * *$ \\
\hline MH-97 × Parwaz-94 & $-1.83^{\mathrm{NS}}$ & $-8.56^{\mathrm{NS}}$ & $5.72^{\mathrm{NS}}$ & $2.80^{\mathrm{NS}}$ & $-3.40^{\mathrm{NS}}$ & $-7.02^{\mathrm{NS}}$ & $\begin{array}{c}- \\
3.89^{\mathrm{NS}} \\
\end{array}$ & $5.23^{\mathrm{NS}}$ & $-4.70^{\mathrm{NS}}$ & $-6.25^{\mathrm{NS}}$ & $-25.93^{\mathrm{NS}}$ & $-40.00^{\mathrm{NS}}$ & $\begin{array}{c}- \\
12.88^{* *} \\
\end{array}$ & $-16.31 * *$ \\
\hline MH-97 × Iqbal-2000 & $-6.64^{\mathrm{NS}}$ & $-20.09 * *$ & $-0.76^{\mathrm{NS}}$ & $-1.98^{\mathrm{NS}}$ & $15.47 * *$ & $9.15^{*}$ & $-\overline{-}$ & $\begin{array}{c}- \\
6.69^{\mathrm{NS}}\end{array}$ & $-16.71 * *$ & $22.88 * *$ & $-81.67 * *$ & $-88.42 * *$ & -0.94 & $-12.56^{*}$ \\
\hline MH-97 × Uqab-2000 & $-3.88^{\mathrm{NS}}$ & $-9.14^{\mathrm{NS}}$ & $8.05 *$ & $1.04^{\mathrm{NS}}$ & $-11.56^{*}$ & $17.99 * *$ & $-\overline{-}$ & $6.94^{\mathrm{NS}}$ & $0.54^{\mathrm{NS}}$ & $-7.32^{\mathrm{NS}}$ & $-40.00^{\mathrm{NS}}$ & $-40.00^{\mathrm{NS}}$ & -7.00 & $-14.39 * *$ \\
\hline MH-97 × 4072 & $16.53 * *$ & $9.95^{\mathrm{NS}}$ & $9.89 * *$ & $0.01^{\mathrm{NS}}$ & $6.14^{\mathrm{NS}}$ & $6.02^{\mathrm{NS}}$ & $0.73^{\mathrm{NS}}$ & $0.88^{-}$ & $2.15^{\mathrm{NS}}$ & $-10.39 *$ & $97.18^{\mathrm{NS}}$ & $40.00^{\mathrm{NS}}$ & -3.99 & $-10.99 *$ \\
\hline MH-97 × Punjab-96 & $1.93^{\mathrm{NS}}$ & $-12.12 *$ & $2.60^{\mathrm{NS}}$ & $-0.37^{\mathrm{NS}}$ & $-8.80^{\mathrm{NS}}$ & $-13.17 *$ & $0.48^{\mathrm{NS}}$ & $\begin{array}{c}- \\
1.10^{\mathrm{NS}} \\
\end{array}$ & $25.66 * *$ & $25.45 * *$ & $24.00^{\mathrm{NS}}$ & $-24.00^{\mathrm{NS}}$ & $\begin{array}{c}- \\
17.41 * * \\
\end{array}$ & $-29.57 * *$ \\
\hline $4072 \times$ Shahkar-95 & $-1.65^{\mathrm{NS}}$ & $-18.12 * *$ & $-5.25^{\mathrm{NS}}$ & $-9.23 * *$ & $-0.92^{\mathrm{NS}}$ & $2.41^{\mathrm{NS}}$ & $\begin{array}{c}- \\
2.20^{\mathrm{NS}}\end{array}$ & $\begin{array}{c}- \\
2.57^{\mathrm{NS}}\end{array}$ & $2.98^{\mathrm{NS}}$ & $-7.64^{\mathrm{NS}}$ & -12.09 & $-42.86^{\mathrm{NS}}$ & -8.10 & $-12.02 *$ \\
\hline $4072 \times$ Parwaz-94 & $-6.57^{\mathrm{NS}}$ & $-17.53 * *$ & $17.47 * *$ & $10.26^{* *}$ & $-9.60^{\mathrm{NS}}$ & $-12.90 *$ & $5.87^{\mathrm{NS}}$ & $4.23^{\mathrm{NS}}$ & $-9.36^{\mathrm{NS}}$ & $19.38 * *$ & $-3.85^{\mathrm{NS}}$ & $-19.35^{\mathrm{NS}}$ & 4.32 & 0.52 \\
\hline $4072 \times$ Iqbal- 2000 & $-15.18 * *$ & $-23.55 * *$ & $0.63^{\mathrm{NS}}$ & $-7.38 *$ & $7.70^{\mathrm{NS}}$ & $1.70^{\mathrm{NS}}$ & $4.99^{\mathrm{NS}}$ & $2.23^{\mathrm{NS}}$ & $-1.18^{\mathrm{NS}}$ & $-6.85^{\mathrm{NS}}$ & $-71.56 * *$ & $-84.21 * *$ & $13.01 *$ & 7.14 \\
\hline $4072 \times$ Uqab-2000 & $-4.84 *$ & $-14.82 *$ & $7.58 *$ & $4.48^{\mathrm{NS}}$ & $-2.42^{\mathrm{NS}}$ & $-9.43^{\mathrm{NS}}$ & $3.59^{\mathrm{NS}}$ & $2.54^{-} \mathrm{NS}$ & $-11.97 * *$ & $27.99 * *$ & $43.66^{\mathrm{NS}}$ & $2.00^{\mathrm{NS}}$ & 9.58 & 8.74 \\
\hline $4072 \times$ MH-97 & $-15.47 * *$ & $-20.24 * *$ & $1.91^{\mathrm{NS}}$ & $-7.25^{*}$ & $-6.09^{\mathrm{NS}}$ & $-6.19^{\mathrm{NS}}$ & $0.61^{\mathrm{NS}}$ & $0.46^{\mathrm{NS}}$ & $-6.88^{\mathrm{NS}}$ & $18.33^{* * *}$ & $-38.03^{\mathrm{NS}}$ & $-56.00^{\mathrm{NS}}$ & -2.74 & -9.83 \\
\hline $4072 \times$ Punjab-96 & $-10.16^{* *}$ & $-18.40 * *$ & $8.36^{*}$ & $1.36^{\mathrm{NS}}$ & $-8.18^{\mathrm{NS}}$ & $\begin{array}{c}- \\
12.66^{* * *} \\
\end{array}$ & $\begin{array}{c}- \\
3.65^{\mathrm{NS}} \\
\end{array}$ & $5.03^{\mathrm{NS}}$ & $2.36^{\mathrm{NS}}$ & $-10.08^{*}$ & $40.85^{\mathrm{NS}}$ & $0.00^{\mathrm{NS}}$ & $21.95 * *$ & 11.33 \\
\hline $\begin{array}{c}\text { Punjab-96 } \times \text { Shahkar- } \\
95 \\
\end{array}$ & $-22.20 * *$ & $-39.98 * *$ & $0.05^{\mathrm{NS}}$ & $-2.41^{\mathrm{NS}}$ & $-6.08^{\mathrm{NS}}$ & -9.36 & $\begin{array}{c}- \\
1.01^{\mathrm{NS}} \\
\end{array}$ & $\begin{array}{c}- \\
2.79^{\mathrm{NS}} \\
\end{array}$ & $-1.34^{\mathrm{NS}}$ & $-3.63^{\mathrm{NS}}$ & $-30.00^{\mathrm{NS}}$ & $-40.00^{\mathrm{NS}}$ & 4.48 & -8.31 \\
\hline Punjab-96 × Parwaz-94 & $-15.88 *$ & $-32.66 * *$ & $3.70^{\mathrm{NS}}$ & $3.32^{\mathrm{NS}}$ & $-10.18^{*}$ & $\begin{array}{c}- \\
17.53 * *\end{array}$ & $0.33^{\mathrm{NS}}$ & $\begin{array}{c}- \\
2.60^{\mathrm{NS}}\end{array}$ & $-9.40^{\mathrm{NS}}$ & $-10.70^{\mathrm{NS}}$ & $-1.23^{\mathrm{NS}}$ & $-20.00^{\mathrm{NS}}$ & 5.36 & -6.98 \\
\hline $\begin{array}{c}\text { Punjab-96 } \times \text { Iqbal- } \\
2000 \\
\end{array}$ & $-1.46^{\mathrm{NS}}$ & $-2.30^{\mathrm{NS}}$ & $-8.90 *$ & $\begin{array}{c}- \\
10.47 * * \\
\end{array}$ & $-3.52^{\mathrm{NS}}$ & $-4.25^{\mathrm{NS}}$ & $\begin{array}{c}- \\
3.72^{\mathrm{NS}} \\
\end{array}$ & $-7.56^{*}$ & $-6.14 *$ & $\begin{array}{c}- \\
12.96^{* *} \\
\end{array}$ & $-66.67 * *$ & $-78.95 * *$ & 6.40 & 2.23 \\
\hline $\begin{array}{c}\text { Punjab-96 } \times \text { Uqab- } \\
2000 \\
\end{array}$ & $-5.14^{\mathrm{NS}}$ & $-21.97 * *$ & $5.08^{\mathrm{NS}}$ & $1.09^{\mathrm{NS}}$ & $-7.72^{\mathrm{NS}}$ & $18.21 * *$ & $5.20^{\mathrm{NS}}$ & $\begin{array}{c}- \\
2.36^{\mathrm{NS}} \\
\end{array}$ & $-15.97 * *$ & $\begin{array}{c}- \\
22.66^{* *} \\
\end{array}$ & $-30.00^{\mathrm{NS}}$ & $-30.00^{\mathrm{NS}}$ & 6.25 & -2.32 \\
\hline Punjab-96 × MH-97 & $-0.32^{\mathrm{NS}}$ & $-14.05 * *$ & $-7.11^{*}$ & $-9.81 * *$ & $8.56^{\mathrm{NS}}$ & $3.36^{\mathrm{NS}}$ & $2.6^{-} \mathrm{NS}$ & $\begin{array}{c}- \\
4.16^{\mathrm{NS}} \\
\end{array}$ & $-21.72 * *$ & $\begin{array}{c}- \\
21.85^{* *} \\
\end{array}$ & $40.00^{\mathrm{NS}}$ & $40.00^{\mathrm{NS}}$ & 8.90 & -7.14 \\
\hline Punjab-96 × 4072 & $-6.89^{\mathrm{NS}}$ & $-15.44 * *$ & $3.75^{\mathrm{NS}}$ & $-2.95^{\mathrm{NS}}$ & $-1.30^{\mathrm{NS}}$ & $-6.12^{\mathrm{NS}}$ & $1.66^{\mathrm{NS}}$ & $0.20^{\mathrm{NS}}$ & $7.10^{\mathrm{NS}}$ & $\begin{array}{c}- \\
18.40 * *\end{array}$ & $-15.49^{\mathrm{NS}}$ & $-40.00^{\mathrm{NS}}$ & -5.05 & $-13.31^{*}$ \\
\hline
\end{tabular}

**, Significant at $P \leq 0.01 ; *$, Significant at $P \leq 0.05$; ns, Non-significant at $P>0.05$. 


\section{Discussion}

During this study, a program was initiated to observe genetic differences between wheat varieties and their cross combinations for flag leaf area and its related traits. Through analysis of variance (ANOVA; Table 1), it was found that all genotypes were different significantly for all plant traits except for stomatal size. These results are in agreement with other researchers as they also found dissimilarity between genotypes for plant yield and other traits in wheat varieties [19, 25-28]. In crop plants genetic variability is an important source for creating new genetic combinations with maximum ability to produce higher yield [29-32]. The supremacy of hybrids over better parent is important to find out option for utilization of heterosis on a commercial scale and recognize specific combinations for producing transgressive segregants of premier quality. In this study, as parent wheat varieties are highly adapted varieties so heterosis and heterobeltosis played an important role for improvement in yield. For future wheat breeding programs, study for the extent of heterosis is important. Leaf and its related traits play critical role in survival of the plant both under optimum and deficient moisture levels. The increase in area of flag leaf in wheat is important for high yield production. Several researchers working on wheat concluded that positive heterosis for flag leaf area ultimately can produce higher grain yield, as flag leaves have significant contribution in production of photosynthates, which are ultimately translocated to grain [18]. Maximum midparent heterosis in hygrophilic colloids was found to be high $(-81.67 \%)$ among the plant traits during the study of seven parental complete diallel analysis [19] which showed that hygrophilic colloids played an important role in yield contribution and stability of yield. The negative sign showed there would be higher yield with decreasing amount of hygrophilic colloids in cross hybrids [30]. Epidermal cell size is also a vital plant trait contributing to high yield and yield stability. Maximum positive flag leaf area (16.53) and leaf venation (9.89) were found in MH-97 $\times 4072$ followed by Uqab-2000 $\times$ Punjab-96 with hygrophilic colloids (-28.00) and other traits while other flag leaf related traits like positive leaf venation (8.36), epidermal cell size (2.36) and stomatal frequency (-8.18), stomatal size (-3.65), was found in $4072 \times$ Punjab-96. Conclusion

The results indicated that the plant traits studied in this experiment were affected on the efficiency of plants to grow well with the limited use of water if a shortage of water occur as these traits enable the plants to make them water use efficient. Crosse MH$97 \times 4072$ showed best performance followed by Uqab-2000 $\times$ Punjab-96 and $4072 \times$ Punjab-96. Considering performance of these crosses, they can be used as promising lines for further breeding program. Due to better characteristics of flag leaf traits these crosses have potential for the development of drought resistant through efficient water utilization which is the key for plant survival under normal as well as under adverse environmental conditions.

\section{Authors' contributions}

Envisioned the project, designed and performed the experiment: S Mahpara, S Hussain \& J Iqbal. Performed statistical analysis: S Mahpara, MIA Rehmani, JS Dar, M.K. Qureshi \& MA Shehzad. Wrote and edited the paper: S. Mahpara, MIA Rehmani, M.K. Qureshi \& JS Dar. All authors read the final draft.

\section{References}

1. Akhtar S, Hussain M, Hassan S \& Nadeem M (2015). Economics and dependence of wheat productivity on farm size in Southern Punjab. J Environ Agric Sci 2: 4. 
2. Dogar $\mathrm{MH}$, Akhtar $\mathrm{S} \&$ Hussain $M$ (2016). Factors affecting yield gap in different wheat varieties in Southern Punjab. J Environ Agric Sci 7: 42-47.

3. Rehmani MIA, Fareed MF, Alvi AM, Ibrahim M, Hussain N, Hussain S, Iqbal J, Bashir MA \& Nawaz H (2016). Delayed wheat (Triticum aestivum L.) cultivation and role of diverse seeding rates and row spacings under semiarid agro-climatic situations. Pure Appl Biol 5(1): 72-84.

4. Government of Pakistan (2016). Economic Survey of Pakistan 2015-16, Ministry of Finance, Islamabad, Pakistan.

5. Saleem M, Chowdhry MA, Kashif M \& Khaliq M (2005). Inheritance pattern of plant height, grain yield and some leaf characteristics of spring wheat. Int $J$ Agric Biol 6: 1015-1018.

6. Kashif M \& Khaliq I (2004). Heritability, correlation and path coefficient analysis for some metric traits in wheat. Int $J$ Agric Biol 6(1): 138-142.

7. Mahmood MT, Khan SJ, Ali I, Hussain S, Shah SAS, Sadiq MA, Ashfaq M \& Ali $F$ (2016). Estimation of genetic variation for agro-economic traits in wheat (Triticum aestivum L.). J Environ Agric Sci 9: 10-14.

8. Dere F \& Yildirim MB (2006). Inheritance of grain yield per plant, flag leaf width and length in an $8 \times 8$ diallel cross population of bread wheat ( $\mathrm{T}$. aestivum L.). Turkish J Agric 30: 339345.

9. Wall GW, Kimball BA, White JW \& Ottman MJ (2011). Gas exchange and water relations of spring wheat under full-season infrared warming. Global Change Biol 17(6): 2113-2133.

10. Shah NH \& Paulsen GM (2003). Interaction of drought and high temperature on photosynthesis and grain-filling of wheat. Plant Soil 257(1): 219-226.
11. Schnable PS \& Springer NM (2013). Progress towards understanding heterosis in crop plants. Annu Rev Plant Biol 64: 13.1-13.18.

12. Shull, GH (1952) Beginnings of the heterosis concept. In: GOWEN, J.W. (Ed.) Heterosis: a record of researches directed toward explaining and utilizing the vigor of hybrids. Ames: Iowa State College Press p.14-48.

13. Falconer DS \& Mackay TFC (1996). Introduction to Quantitative Genetics. 4th ed, England: Longman Essay.

14. Freeman GF (1919). Heredity of quantitative characters in wheat. Genetics 4: 1-93.

15. Briggle LW (1963). Heterosis in wheat: a review. Crop Sci 3: 407-412.

16. Thomas N, Marker S, Lal GM \& Dayal A (2017). Study of heterosis for grain yield and its components in wheat (Triticum aestivum) over normal and heat stress condition. $J$ Pharm Phytochem 6(4): 824-830.

17. Mahpara S, Ali Z, Rehmani MIA, Iqbal J $\&$ Shafiq MR (2017). Studies of genetic and combining ability analysis for some physio-morphological traits in spring wheat using $7 \times 7$ diallel crosses. Int $J$ Agric Appl Sci 9(1): 33-40.

18. Jatoi WA, Baloch MJ, Khan NU, Munir M, Khakwani AA, Vesar NF, Panhwar SA \& Gul S (2014). Heterosis for yield and physiological traits in wheat under water stress conditions. J Animal Plant Sci 24(1): 252-261.

19. Mahpara S (2008). Biometrical analysis of important plant attributes in spring wheat, Ph.D. Dissertation, Department of Plant Breeding and Genetics, University of Agriculture, Faisalabad, Pakistan.

20. Steel RGD, Torrie JH \& Discky DA (1997). Principles and Procedures of Statistics: A Biometrical Approach. 3rd ed, McGraw Hill Book Co., New York. 
21. Matzingar DF, Mann TJ \& Cockerham CC (1962). Diallel crosses in Nicotiana tabaccum. Crop Sci 2: 383-386.

22. Hayman BI (1954). The theory of analysis of diallel crosses. Genetics 39: 739-809.

23. Hayman BI (1954). The analysis of variance of diallel crosses. Biometrics 10: $235-245$.

24. Jinks JL (1954). The analysis of continuous variation in diallel crosses of Nicotiana rustica L. vatieties. Genetics 39: 767-788.

25. Ambreen A, Chowdhry MA, Khaliq I \& Ahmad R (2002). Genetic determination for some drought related leaf traits in bread wheat. Asian J Pl Sci 1(3): 232234.

26. Singh I \& Paroda RS (1987). Partial diallel analysis for combining ability in wheat. Indian $J$ Genet Pantl Breed 47(1): 1-5.

27. Singh KN (1988). Combining ability in wheat in normal and sodic soils. Indian J Genet Plant Breed 48: 99-102.
28. Menon U \& Sharma SN (1997). Inheritance studies for yield and yield component traits in bread wheat over the environment. Wheat Inf Serv 80: 1-5.

29. Mahpara S, Ali Z, Farooq J, Hussain S \& Bibi R (2015). Heterosis and heterobeltiosis analysis for spike and its related attiributes in different wheat crosses. Pak J Nutri 14(7): 396.

30. Hei N, Hussein S \& Laing M (2016). Heterosis and combining ability analysis of slow rusting stem rust resistance and yield and related traits in bread wheat. Euphytica 207(3): 501-514.

31. Yücel C, Baloch FS \& Özkan H (2009). Genetic analysis of some physical properties of bread wheat grain (Triticum aestivum L. em Thell). Turkish J Agric Forest 33(6): 525-535.

32. Yücel C, Baloch FS, Hatipoğlu R \& Özkan H (2011). Genetic analysis of preharvest sprouting tolerance in bread wheat (Triticum aestivum L. emend. Thell.). Turkish J Agric Forest 35(1): 922. 